\title{
Pretratamiento Alcalino de Pasto Elefante (Pennisetum sp) y King Grass (Pennisetum hybridum) Cultivados en Colombia para la Producción de Bioetanol
}

\author{
Eliana M. Cardona ${ }^{(1)}$, Jorge A. Rios ${ }^{(1)}$, Juan D. Peña ${ }^{(2)}$ y Luis A. Rios ${ }^{(1) *}$ \\ (1) Depto. de Ingeniería Química, Facultad de Ingeniería, Universidad de Antioquia UdeA, \\ Calle 70 No. 52-21, Medellín-Colombia. (e-mail: larios@udea.edu.co) \\ (2) Empresas Públicas de Medellín E.S.P., Medellín-Colombia. (e-mail: Juan.Pena.Alvarez@epm.com.co) \\ * Autor a quien debe ser dirigida la correspondencia.
}

Recibido Mar. 12, 2013; Aceptado Abr. 29, 2013; Versión final recibida May. 09, 2013

\begin{abstract}
Resumen
Se evaluó el efecto de diferentes condiciones del pretratamiento con hidróxido de sodio $(\mathrm{NaOH})$ en la recuperación de la fracción celulósica, remoción de lignina y producción de etanol mediante fermentación y sacarificación simultáneas de los pastos elefante y king grass (Pennisetum purpureum and Pennisetum hybridum). Estos pastos son materias primas potenciales para la obtención de bioetanol a partir de la fracción celulósica. Los resultados obtenidos en producción de etanol muestran que bajo condiciones de pretratamiento de $120^{\circ} \mathrm{C}, 60$ minutos, $\mathrm{NaOH}$ al $2 \%$ (w/w) y una relación líquido a sólido de 20 (w/w) se obtienen las más altas concentraciones de etanol: $27.7 \mathrm{~g} / \mathrm{L}$ para king grass y $26.1 \mathrm{~g} / \mathrm{L}$ para pasto elefante en 24 horas de fermentación. Además, bajo las condiciones evaluadas se pudo observar remociones de lignina de $88.4 \%$ y $94.0 \%$ para pasto elefante y pasto king grass respectivamente. La etapa de desintoxicación permite eliminar inhibidores formados durante el pretratamiento, los cuales afectan la hidrólisis y fermentación.
\end{abstract}

Palabras clave: remoción de lignina, king grass, pasto elefante, rendimiento a etanol, azucares reductores

\section{Alkaline Pretreatment of Elephant Grass (Pennisetum Sp) and King Grass (Pennisetum Hybridum) Cultured in Colombia for Ethanol Production}

\begin{abstract}
The effect of different alkaline pretreatment conditions with sodium hydroxide $(\mathrm{NaOH})$ on the recuperation of cellulosic fraction, lignin removal and ethanol production was evaluated through simultaneous saccharification and fermentation of elephant grass and king grass ((Pennisetum purpureum and Pennisetum hybridum). These types of grass are potential raw materials for bioethanol production from cellulosic fraction. Results obtained in ethanol production show that under pretreatment conditions of $120^{\circ} \mathrm{C}$, 60 minutes, $2 \%(\mathrm{w} / \mathrm{w})$ of $\mathrm{NaOH}$ and a liquid to solid ratio of $20(\mathrm{w} / \mathrm{w})$, the highest ethanol concentrations are obtained: $27.7 \mathrm{~g} / \mathrm{L}$ and $26.1 \mathrm{~g} / \mathrm{L}$ for king grass and elephant grass respectively, in 24 hours of fermentation. Furthermore, under the evaluated conditions it was observed that lignin removal was $88.4 \%$ for elephant grass and $94.0 \%$ for king grass. The detoxification stage eliminates inhibitors formed during pretreatment, which affects the hydrolysis and fermentation.
\end{abstract}

Keywords: lignin removal, king grass, elephant grass, ethanol yield, reducing sugars 


\section{INTRODUCCIÓN}

La mayoría de países tropicales, como Colombia, presentan un gran potencial en cuanto a la disponibilidad y variedad de biomasa lignocelulósica debido a su alta radiación solar, a sus diferentes pisos térmicos y a su biodiversidad. Estas ventajas le permiten una fácil adaptación de las diferentes especies para el desarrollo de cultivos energéticos como pastos y forrajes; dentro de los cuales, los pastos elefante (Pennisetum $s p$ ) y king grass (Pennisetum hybridum) pertenecientes a la familia Poaceae, subfamilia Panicoideae, presentan altos rendimientos de producción de materia seca por hectárea al año, entre 40-50 toneladas para pasto elefante y entre 60-80 toneladas para king grass bajo condiciones óptimas de crecimiento y manejo (Mármol, 1998; González et al., 2011;Cardona et al., 2012).

Por su composición química, la biomasa lignocelulósica es muy diferente de los productos con alto contenido de azúcares o almidón. La estructura de estos materiales, compuesta fundamentalmente por celulosa, hemicelulosa y lignina, hace que los procesos para la obtención de biocombustibles deban ajustarse de acuerdo a las características de estos componentes, haciendo necesaria la implementación de una etapa previa de pretratamiento para poder conseguir fracciones hidrolizables para la obtención de azúcares que posteriormente puedan ser fermentadas (Abril y Abril, 2008). La etapa de pretratamiento, es quizás más importante y la de mayor impacto sobre las demás etapas del proceso, como la hidrólisis enzimática, fermentación, manejo de aguas residuales y corrientes de proceso, en términos de digestibilidad de la celulosa, toxicidad en la fermentación, requerimientos de potencia para la agitación, demanda de energía en procesos posteriores y demanda de tratamiento de aguas (Galbe y Zacchi, 2007).

La química del pretratamiento puede involucrar la modificación química, despolimerización y/o solvatación, redistribución física de la lignina y hemicelulosa, y potencialmente alterar la cristalinidad de la celulosa (Banerjee et al., 2011). Diferentes tipos de pretratamientos físicos, químicos, fisicoquímicos y biológicos de la biomasa lignocelulósica han sido ampliamente estudiados para mejorar la producción de etanol. Todos estos métodos pueden hacer la lignocelulosa más accesible a la hidrólisis enzimática, donde la cristalinidad de la celulosa, el área superficial accesible, y la disponibilidad de lignina y hemicelulosa son los principales factores que afectan la hidrólisis. Sobre los diferentes métodos de pretratamiento, los químicos y termoquímicos son los más comúnmente efectivos e incluyen las tecnologías más promisorias para aplicaciones industriales (Alvira et al., 2010; Taherzadeh y Karimi, 2008).

Se han reportado diferentes tipos de pretratamientos bajo diferentes condiciones, con el fin de mejorar la digestibilidad y fermentabilidad de distintas variedades de pastos como bermuda, switchgrass, napiergrass y silvergrass (Anderson et al., 2005; Digman et al., 2010; Isci et al., 2007; Keshwani y Cheng, 2009; López et al., 2010; Sun y Cheng, 2005; Wang et al.,2010a; Woodward, 2006). El pretratamiento ácido a $121^{\circ} \mathrm{C}$, de pasto bermuda con una carga de sólido de 10\%, mostró una conversión a glucosa de $70 \%$ con una producción de azúcares reductores totales de $204,1 \mathrm{mg} / \mathrm{g}$ de biomasa en 48 horas de hidrólisis enzimática, para una concentración de ácido sulfúrico de $1.2 \%$ y 60 minutos de pretratamiento (Sun y Cheng, 2005). Así mismo, el pretratamiento con ácido sulfúrico diluido de silvergrass a $121^{\circ} \mathrm{C}$, por 30 minutos, permitió una alta recuperación de xilosa (70-75\%) en comparación con la cascarilla de arroz y el bagazo. El hidrolizado de silvergrass tuvo además, un alto nivel de fermentabilidad en comparación al bagazo, debido que se formó una menor cantidad de ácido acético, obteniendo un rendimiento de $64,3 \%$ del máximo teórico en 48 horas de fermentación (Guo et al., 2008). También se ha evaluado el pretratamiento alcalino de pasto Bermuda, empleando $\mathrm{NaOH}$ y $\mathrm{Ca}(\mathrm{OH})_{2}$ para mejorar la recuperación de azúcares fermentables. El estudio mostró que el $\mathrm{NaOH}$ es más eficiente que el $\mathrm{Ca}(\mathrm{OH})_{2}$ a $121^{\circ} \mathrm{C}$ para mejorar el rendimiento de azúcares reductores, alcanzando aproximadamente $86 \%$ del máximo teórico (aproximadamente $500 \mathrm{mg}$ de azucares reductores totales/ g de biomasa) (Wang et al., 2008). Otros estudios reportan una remoción de lignina hasta del $86 \%$ y un rendimiento total a azucares reductores de $71 \%$ del máximo teórico $(440 \mathrm{mg}$ de azucares reductores totales/ $\mathrm{g}$ de biomasa) para unas condiciones óptimas de pretratamiento de pasto bermuda de 15 minutos y $0.75 \%$ de $\mathrm{NaOH}$ a $121^{\circ} \mathrm{C}$, sin evaluación de la fermentabilidad del material hidrolizado (Wang et al., 2010b).

Hasta el momento existen pocos reportes sobre los estudios del efecto de diferentes pretratamientos en la hidrólisis y fermentabilidad de los pastos elefante y king grass, los cuales están enfocados específicamente en el pretratamiento y fermentabilidad de variedades del pasto Penisetum purpureum (Anderson et al., 2008; Anderson et al., 2005; Brandon et al., 2011; Segura et al., 2008; Tran et al., 2011; Yoshida et al., 2008). En cuanto al pretratamiento, se reporta el pretratamiento enzimático (celulasa + estearasa) de ésta variedad de pasto con la liberación de $113 \mathrm{mg}$ de azúcares por gramo de biomasa empleada (Anderson et al., 2005) y en el pretratamiento biológico para la deslignificación selectiva de una especie colombiana de penisetum $s p$. usando basidiomicetos ligninolíticos, se alcanzaron disminuciones en el contenido de lignina en detergente ácido de $55.9 \%$ a $10.7 \%$ con ganoderma spp.(Segura et al., 2008). En cuanto a la fermentabilidad se reportan rendimientos a etanol de $47,5 \mathrm{mg} / \mathrm{g}$ de biomasa usando la cepa de Klebsiella 
oxytoca THLC0409 y rendimientos de 107mg y $97 \mathrm{mg}$ por gramo de biomasa usando la cepa Saccharomyces cerevisiae $\mathrm{D}_{5} \mathrm{~A}$ para dos genotipos diferentes de pasto elefante (Anderson et al., 2008). Así mismo, se reporta la fermentación de pentosas y hexosas del pasto penisetum sin pretratar empleando Saccharomyces cerevisiae NBRC2044 y E. coli K011 logrando la producción de $113 \mathrm{mg}$ de etanol/g de biomasa para el SSF de las hexosas y $31,4 \mathrm{mg}$ de etanol/g de biomasa a partir la fermentación de pentosas (Yasuda et al., 2012).

La producción a mayor escala, incluso comercial, de etanol lignocelulósico ya es una realidad. Actualmente existen numerosas plantas a nivel piloto, demostrativo y comercial para la producción de etanol de segunda generación, en países como Estados Unidos, Alemania, Francia, España, Suiza, y Dinamarca. Algunas de las plantas existentes como la DTU Biogasol, Inbicon DONG Energy, Sekab, Biogasol, entre otras, tienen capacidades de producción de etanol entre 2 -1100 t/año para plantas piloto, entre 2840 - 50000 t/año para plantas demostrativas, y desde 120000 t/año para plantas comerciales; a partir de sustratos como residuos maderables, switchgrass, paja de trigo, bagazo de caña, residuos del cultivo de maíz, y otros diferentes tipos de residuos agrícolas (Gnansounou, 2010).

En este artículo se presenta el efecto de diferentes condiciones del pretratamiento alcalino en la hidrólisis enzimática y fermentabilidad de los pastos elefante y king grass cultivados en el oriente del departamento de Antioquia, los cuales son materias primas alternativas que presentan un gran potencial para la explotación en el campo de la producción de biocombustibles.

\section{DESARROLLO EXPERIMENTAL}

\section{Materiales}

Los pastos elefante y king grass fueron adquiridos en granjas localizadas en el oriente del departamento de Antioquia, Colombia. Los pastos fueron secados al aire para facilitar su manipulación y transporte, y luego fueron sometidos a un proceso de disminución de tamaño usando un molino de cuchillas para material vegetal. El material molido obtuvo un tamaño de partícula menor de $3 \mathrm{~mm}$ y fue secado nuevamente a $60^{\circ} \mathrm{C}$ hasta alcanzar un contenido de humedad inferior al $10 \% \mathrm{y}$, posteriormente fue almacenado en cajas herméticas hasta su uso.

\section{Optimización deslignificación alcalina con $\mathrm{NaOH}$}

Se pesó 50,0 g de material, se llevó al reactor y se adicionó una solución de soda cáustica de acuerdo a la relación sólido/líquido y tiempo establecidos en el diseño mostrado en la tabla 1. El pretratamiento alcalino fue llevado a cabo en una autoclave para los experimentos que requerían temperaturas mayores a $100{ }^{\circ} \mathrm{C}, \mathrm{y}$ en reactores con agitación mecánica para los experimentos que requerían condiciones menos drásticas de esta variable. Completado el tiempo se enfrío hasta temperatura ambiente, para luego filtrar y separar el material del licor negro; el sólido se lavó con agua hasta pH neutro para luego secarlo y almacenarlo en nevera para su posterior uso. Con el fin de establecer las condiciones óptimas del pretratamiento alcalino dentro de los rangos seleccionados para cada una de las variables, se evaluó la fermentabilidad del material pretratado. Las condiciones establecidas para la evaluación del pretratamiento en los pastos elefante y king grass fueron: temperatura entre 80 y $120^{\circ} \mathrm{C}$, tiempo de residencia entre 30 y 180 minutos, relación sólido/líquido (w/w) 1:15 a 1:20, y concentración de $\mathrm{NaOH}$ entre $1 \%$ y $2 \%$ (w/w). El sólido obtenido fue neutralizado con una solución de $\mathrm{HCl}$ y lavado con $600 \mathrm{~mL}$ agua destilada. Bajo estos rangos de condiciones de operación, se realizó un diseño experimental tipo Draper - Lin con tres puntos centrales, que permitiera optimizar las condiciones de pretratamiento con el fin de maximizar el rendimiento a etanol, mostrado en la tabla 1. Los datos que se muestran en las figuras y tablas corresponden al valor promedio de experimentos por triplicado con una desviación estándar relativa menor del $5 \%$ en todos los casos.

\section{Hidrólisis Enzimática}

La hidrólisis de los materiales pretratados se llevó a cabo usando la enzima comercial Accellerase 1500 de Genecor, con una carga de 30FPU/g de biomasa. Para la hidrólisis se montaron los sistemas en un shaker a una temperatura de $50^{\circ} \mathrm{C}, 180 \mathrm{rpm}$ y pH 4.8 para amortiguar cambios en acidez o alcalinidad del sistema, y se monitoreo periódicamente para determinar la producción de azúcares reductores.

\section{Sacarificación y fermentación simultánea (SSF)}

La hidrólisis y fermentación simultánea (SSF) se llevó a cabo usando la enzima Accellerase 1500 de Genecor y la levadura comercial Saccharomyces cerevisiae Ethanol Red. Después de transcurridas 12 horas de hidrólisis enzimática a $50^{\circ} \mathrm{C}, 180 \mathrm{rpm}$ y $\mathrm{pH} 4.8$, se inocula una cantidad de levadura que permita 
obtener una concentración de $2 \mathrm{~g} / \mathrm{L}$ de levadura seca en el medio de fermentación, se disminuye la temperatura a $37^{\circ} \mathrm{C}$, la agitación a $150 \mathrm{rpm}$ y se acopla una trampa de agua para el $\mathrm{CO}_{2}$ liberado durante la fermentación. El seguimiento de la fermentación fue realizado por pérdida de peso y la concentración final de etanol en la suspensión fue determinada por cromatografía gaseosa acoplada a un sistema de micro extracción en fase sólida (SPME). La determinación del rendimiento máximo a etanol a partir de estos materiales se determinó con base en la cantidad de celulosa contenida en el material.

Tabla 1: Diseño experimental para la optimización del pretratamiento alcalino de pastos elefante y king grass.

\begin{tabular}{ccccc}
\hline \multirow{2}{*}{ Experimento } & \multicolumn{3}{c}{ CONDICIONES } \\
\cline { 2 - 4 } & $\mathrm{T}^{\circ} \mathrm{C}$ & $\mathrm{t}(\mathrm{h})$ & $\%$ NaOH $(\mathrm{w} / \mathrm{w})$ & Relación S/L $(\mathrm{w} / \mathrm{w})$ \\
\hline 1 & 100 & 2 & 1,5 & $1: 17,5$ \\
2 & 120 & 1 & 1 & $1: 20$ \\
3 & 100 & 2 & 2,3 & $1: 17,5$ \\
4 & 66,4 & 2 & 1,5 & $1: 17,5$ \\
5 & 100 & 2 & 1,5 & $1: 17,5$ \\
6 & 120 & 3 & 2 & $1: 15$ \\
7 & 120 & 3 & 1 & $1: 20$ \\
8 & 80 & 3 & 1 & $1: 17,5$ \\
9 & 100 & 3,7 & 1,5 & $1: 21,7$ \\
10 & 100 & 2 & 1,5 & $1: 17,5$ \\
11 & 100 & 2 & 1,5 & $1: 17,5$ \\
12 & 100 & 0,7 & $1: 13,3$ \\
13 & 100 & 2 & 1,5 & $1: 20$ \\
14 & 120 & 2 & 2 & $1: 17,5$ \\
16 & 100 & 1 & 1,5 & $1: 20$ \\
18 & 80 & 0,3 & 2 & $1: 15$ \\
19 & 80 & 3 & 2 & $1: 17,5$ \\
\end{tabular}

\section{Análisis}

Los materiales fueron caracterizados siguiendo los protocolos del National Renewable Energy Laboratory (NREL) para la determinación de cenizas, humedad y extractivos en biomasa (Sluiter et al., 2008a; Sluiter et al., 2008b; Sluiter et al., 2008c). Los contenidos de celulosa, hemicelulosa y lignina en los materiales pretratados y sin pretratar se realizaron mediante espectroscopía UV-vis después de la hidrólisis ácida con $72 \% \mathrm{H}_{2} \mathrm{SO}_{4}$ empleando los métodos del ácido 3,5-dinitrosalicílico (DNS) para azúcares reductores y glucosa oxidasa/peroxidasa de BioSystems ${ }^{\circledR}$ para determinación de glucosa.

Los azúcares reductores obtenidos durante la hidrólisis fueron determinados empleando nuevamente los métodos del ácido 3,5-dinitrosalicílico (DNS) y glucosa oxidasa/peroxidasa de BioSystems ${ }^{\circledR}$. El seguimiento de la fermentación se realizó por pérdida de peso del sistema y, por estequiometria, se calculó la conversión teórica de los azúcares obtenidos en la hidrólisis del material en etanol; este SSF fue monitoreado por 26 horas aproximadamente. El etanol producido al final de la fermentación fue determinado por cromatografía de gases en un cromatógrafo Agilent 7890 con detector FID empleando una columna HP-INNOWax y el método de microextracción en fase sólida (SPME) usando una fibra de poliacrilato 85- $\mu$ m. Para determinar los inhibidores como xilitol, ácido succínico, ácido láctico, furfural, hidroximetil furfural, ácido acético y glicerol, formados durante el proceso de pretratamiento, se tomaron muestras de las fracciones líquidas del material pretratado, se filtraron a través de un filtro de $0.25 \mu \mathrm{m}$ y se analizaron mediante HPLC usando una columna Biorad Aminex HPX-87H.

\section{RESULTADOS}

\section{Caracterización del material pretratado}

Las fracciones líquida y sólida del material pretratado fueron caracterizadas empleando las técnicas mencionadas para determinación de celulosa, hemicelulosa y lignina en biomasa, y la realización de los balances de materia, al igual que para el material sin pretratar cuya composición inicial se muestra en la tabla 2. En la tabla 3, se presentan los resultados de la caracterización de la fracción sólida del pretratado, 
así como el porcentaje de sólido que se recupera con respecto a la cantidad alimentada al proceso y los porcentajes de recuperación de celulosa y hemicelulosa, así como también el porcentaje de remoción de lignina, para cada uno de los experimentos planteados (tabla 1) para los pastos king grass y elefante, respectivamente.

Tabla 2: Caracterización de los pastos sin pretratar.

\begin{tabular}{lllllll}
\hline Material & \% Celulosa & \% Hemicelulosa & \% Lignina & \% Cenizas & \% Extractivos & otros \\
\hline Pasto elefante & 22,6 & 20,9 & 19,4 & 11,1 & 9,9 & 16,1 \\
King grass & 23,6 & 21,9 & 15,4 & 14,6 & 16,9 & 7,6 \\
\hline
\end{tabular}

Tabla 3: Caracterización de la fracción sólida de los pastos elefante (PE) y King grass (KG) pretratados con $\mathrm{NaOH}$.

\begin{tabular}{|c|c|c|c|c|c|c|c|c|}
\hline \multirow[t]{2}{*}{ Experimento } & \multicolumn{2}{|c|}{$\begin{array}{c}\% \text { sólido } \\
\text { recuperado }\end{array}$} & \multicolumn{2}{|c|}{$\begin{array}{l}\text { \% Remoción } \\
\text { lignina }\end{array}$} & \multicolumn{2}{|c|}{$\begin{array}{c}\text { \% Recuperación } \\
\text { celulosa }\end{array}$} & \multicolumn{2}{|c|}{$\begin{array}{l}\text { \% Recuperación } \\
\text { Hemicelulosa }\end{array}$} \\
\hline & $\mathrm{PE}$ & $K G$ & $\mathrm{PE}$ & KG & $\mathrm{PE}$ & $K G$ & $\mathrm{PE}$ & KG \\
\hline 1 & 40,4 & 49,1 & 81,5 & 71,6 & 98,1 & 75,4 & 31,4 & 51,7 \\
\hline 2 & 48,5 & 45,2 & 79,3 & 74,7 & 98,9 & 81,7 & 60,5 & 98,4 \\
\hline 3 & 46,9 & 47,5 & 79,9 & 69,1 & 98,3 & 85,6 & 53,1 & 41,9 \\
\hline 4 & 40,5 & 40,6 & 78,2 & 70 & 98,1 & 80,7 & 59,6 & 43 \\
\hline 5 & 51,7 & 44,8 & 83,9 & 76,4 & 97,5 & 92,8 & 46,6 & 22,6 \\
\hline 6 & 49,8 & 48,2 & 77 & 53 & 98,1 & 82,3 & 28,2 & 55,7 \\
\hline 7 & 53,6 & 50,7 & 83,6 & 71,2 & 99 & 82,6 & 81 & 55,8 \\
\hline 8 & 50,5 & 50,2 & 77,9 & 85,9 & 97,6 & 73,3 & 70 & 73,4 \\
\hline 9 & 48,3 & 51,7 & 78,1 & 81,4 & 97,5 & 78,5 & 19,7 & 99,5 \\
\hline 10 & 48,1 & 36,5 & 78,7 & 76,8 & 96,5 & 84,4 & 31,2 & 58,4 \\
\hline 11 & 52,1 & 53,5 & 86,9 & 72,3 & 98,2 & 64,5 & 52,3 & 60,5 \\
\hline 12 & 53,9 & 44,4 & 88,4 & 82,2 & 96,9 & 68,4 & 68,5 & 54,4 \\
\hline 13 & 41,9 & 44,1 & 78,6 & 94 & 97,2 & 61,9 & 57,8 & 48,7 \\
\hline 14 & 51,9 & 56,8 & 79,9 & 76,4 & 97,8 & 67,4 & 82,7 & 28,6 \\
\hline 15 & 44,7 & 42,2 & 81,5 & 80 & 96,8 & 92,7 & 29,7 & 40,1 \\
\hline 16 & 47,8 & 49,9 & 76,2 & 76 & 96,9 & 79,7 & 46,8 & 28,6 \\
\hline 17 & 47,4 & 52,8 & 78,1 & 74,3 & 97,2 & 55,8 & 49,2 & 54,4 \\
\hline 18 & 46,6 & 52,0 & 82,5 & 78,6 & 97,2 & 69,5 & 44,5 & 29,4 \\
\hline 19 & 51,0 & 49,3 & 80,8 & 85,2 & 98,4 & 64,5 & 40,2 & 38,1 \\
\hline
\end{tabular}

Bajo las diferentes condiciones de temperatura, tiempo de residencia, concentración de $\mathrm{NaOH}$ y relación sólido/líquido, empleadas para el pretratamiento de pasto elefante, pudieron obtenerse una recuperación de celulosa en el sólido entre $96,5 \%-99 \%$, y una remoción de lignina entre $76,2 \%-88,4 \%$ con respecto al contenido inicial en el material sin pretratar. En la tabla 3, puede verse que los efectos sobre la remoción de lignina y recuperación de hemicelulosa son muy variables para cada una de las condiciones evaluadas, lo cual se ve reflejado principalmente en los porcentajes de recuperación del sólido, donde se aprecian pérdidas del material entre $40,4 \%$ y $53,9 \%$. Así mismo, para el pasto king grass, se observa (tabla 3 ) que bajo las condiciones experimentales planteadas, entre el $36,5 \%-56,8 \%$ del sólido cargado al inicio del pretratamiento se está solubilizando. En comparación con el pasto elefante, el pasto king grass exhibe un comportamiento más variable en cuanto a la recuperación de celulosa, hemicelulosa, y remoción de lignina, obteniendo recuperaciones de celulosa entre $55,8 \%-92,8 \%$, y logrando remover entre $53,0 \%-94,0 \%$ del contenido de lignina inicialmente presente en el material.

En la fracción líquida obtenida después de cada uno de los ensayos realizados, se cuantificó la cantidad de lignina, celulosa y hemicelulosa, como se muestra en la tabla 4. Estos resultados evidencian que bajo las diferentes condiciones de pretratamiento evaluadas se da una hidrólisis parcial de hemicelulosa y de celulosa en una menor proporción, y además se evidencia una alta concentración de lignina confirmando las altas remociones logradas bajo este pretratamiento. 
Tabla 4: Caracterización de la fracción líquida de los pastos pretratados con $\mathrm{NaOH}$ (porcentajes calculados con base en la fracción de material solubilizada).

\begin{tabular}{|c|c|c|c|c|c|c|}
\hline \multicolumn{4}{|c|}{ King grass } & \multicolumn{3}{|c|}{ Pasto elefante } \\
\hline Experimento & $\%$ Lignina & $\%$ Celulosa & \% Hemicelulosa & $\%$ Lignina & \% Celulosa & \% Hemicelulosa \\
\hline 1 & 21,7 & 0,5 & 2,8 & 39,1 & 1 & 10 \\
\hline 2 & 25,3 & 0,6 & 9,6 & 31,7 & 0,5 & 19 \\
\hline 3 & 26,7 & 1,1 & 2,4 & 33,1 & 0,8 & 21,9 \\
\hline 4 & 26,5 & 0,9 & 15,8 & 37,5 & 1,1 & 15,5 \\
\hline 5 & 26,3 & 0,9 & 11,6 & 31,5 & 1,1 & 18,2 \\
\hline 6 & 23,8 & 1,3 & 19,3 & 30 & 0,9 & 21 \\
\hline 7 & 26,1 & 0,5 & 1,4 & 30,2 & 0,4 & 11,4 \\
\hline 8 & 22 & 1,1 & 24,7 & 29,9 & 1,1 & 19,3 \\
\hline 9 & 25,3 & 0,8 & 2,1 & 31,4 & 1,2 & 21,8 \\
\hline 10 & 32,1 & 1,3 & 20,6 & 31,8 & 1,7 & 27,4 \\
\hline 11 & 22,6 & 0,8 & 17,5 & 32,4 & 0,8 & 14 \\
\hline 12 & 27,7 & 0,8 & 11,7 & 31,8 & 1,3 & 20,2 \\
\hline 13 & 28,5 & 1,1 & 2,7 & 36,4 & 1,5 & 9,7 \\
\hline 14 & 20,7 & 0,7 & 18,3 & 29,9 & 0,9 & 26,8 \\
\hline 15 & 26,4 & 0,7 & 17,4 & 35,4 & 1,6 & 24,4 \\
\hline 16 & 29 & 0,6 & 12,3 & 30,9 & 1,5 & 22,4 \\
\hline 17 & 20,8 & 0,2 & 3,5 & 32 & 1,3 & 20,4 \\
\hline 18 & 15,7 & 0,6 & 14,4 & 34,3 & 1,4 & 21,7 \\
\hline 19 & 24 & 0,9 & 20,4 & 30,7 & 0,7 & 12,9 \\
\hline
\end{tabular}

Se analizaron las muestras de licor negro obtenidas después del pretratamiento mediante HPLC para determinar la presencia de posibles compuestos de degradación o inhibidores formados bajo las condiciones de pretratamiento evaluadas, observándose la formación de ácido acético $(1,62-1,72 \mathrm{~g} / \mathrm{L})$ para el licor proveniente del pretratamiento de king grass, y la presencia de ácido fórmico $(0,08-0,14 \mathrm{~g} / \mathrm{L})$, ácido acético $(1,71-1,96 \mathrm{~g} / \mathrm{L})$ y furfural $(0,003-0,01 \mathrm{~g} / \mathrm{L})$ en el licor proveniente del pretratamiento de pasto elefante.

\section{Hidrólisis enzimática}

La hidrólisis enzimática de los materiales pretratados fue realizada usando la enzima Accellerase 1500 de Genecor a $50^{\circ} \mathrm{C}$ con una carga enzimática de $30 \mathrm{FPU} / \mathrm{g}$ de biomasa y una carga de sólido de $11 \%(\mathrm{w} / \mathrm{v})$. Bajo las diferentes condiciones de pretratamiento evaluadas, pudo obtenerse entre 354,5 y $563,3 \mathrm{mg}$ de azúcares reductores/g de biomasa seca alimentada en la hidrólisis enzimática del pasto elefante, como se muestra en la figura 1. Las mayores producciones de azúcares reductores, se alcanzaron bajo las condiciones más altas de temperatura de pretratamiento $\left(120^{\circ} \mathrm{C}\right)$ logrando más de un $78 \%$ de eficiencia en la conversión de polisacáridos en azucares fermentables. De igual manera, se obtuvieron concentraciones de azúcares reductores a partir de la hidrólisis enzimática de king grass pretratado entre 290,0 y $504,1 \mathrm{mg}$ de azúcares reductores/g de biomasa seca alimentada (figura 1). En este caso no puede establecerse una relación directa entre las variables experimentales y la cantidad de azúcares producidos, sin embargo, la mayor cantidad de azúcares reductores, $515,6 \mathrm{mg}$ de azúcares reductores/g de biomasa para pasto elefante, se obtiene bajo condiciones de $120^{\circ} \mathrm{C}, 3$ horas, $1,0 \%(\mathrm{w} / \mathrm{w}) \mathrm{NaOH}$ y una relación sólido/líquido de $1 \mathrm{~g}$ de material por cada $15,0 \mathrm{~g}$ de solución, y para la hidrólisis de pasto king grass el mayor rendimiento a azúcares reductores $563,3 \mathrm{mg} / \mathrm{g}$ de biomasa se obtiene bajo condiciones de pretratamiento alcalino de 80 ${ }^{\circ} \mathrm{C}$, 1 hora, $2,0 \%(\mathrm{w} / \mathrm{w}) \mathrm{NaOH}$ y una relación sólido/líquido de $1 \mathrm{~g}$ de material por cada $15,0 \mathrm{~g}$ de solución.

Durante la evaluación de las diferentes condiciones de pretratamiento de estos materiales, se logró obtener altas remociones de lignina, sin embargo no puede establecerse una correspondencia entre ésta y la producción de azúcares reductores, lo cual puede deberse a los diferentes efectos del pretratamiento alcalino sobre el material, ocasionando que la lignina remanente bloquee el acceso de la enzima a las partes amorfas de la celulosa que compone las fibras del pasto, afectando así la eficiencia de la hidrólisis enzimática. 


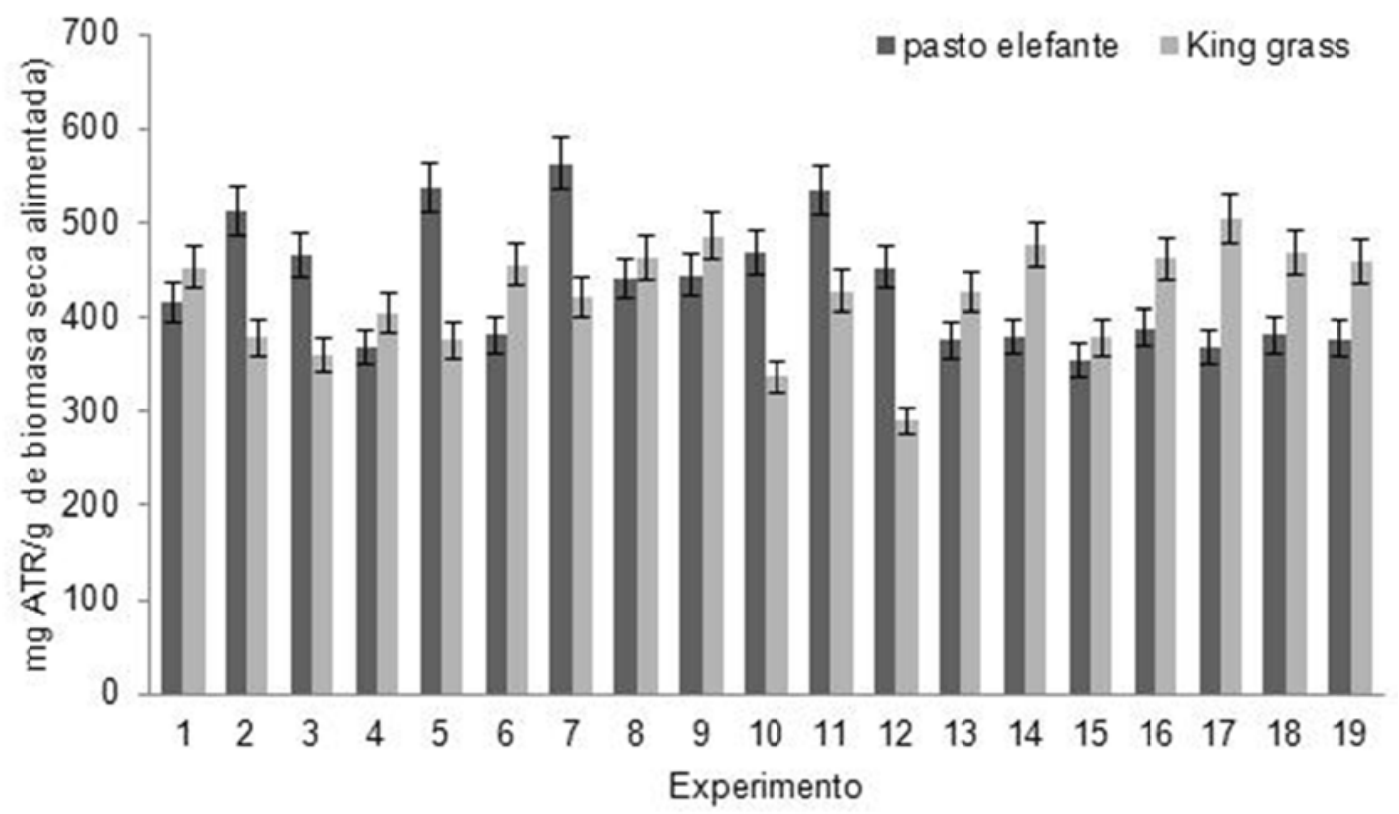

Fig. 1: Producción de azúcares reductores totales para pasto elefante y King grass bajo las diferentes condiciones experimentales.

\section{Sacarificación y fermentación simultaneas (SSF)}

Los resultados obtenidos en la evaluación de la producción de etanol empleando el sistema SSF para los materiales después de ser pretratados bajo diferentes condiciones de la tabla 1, son mostrados en la figura 2 para pasto elefante y pasto king grass, respectivamente. El análisis estadístico realizado a los datos obtenidos para la fermentabilidad de pasto elefante bajo diferentes condiciones de pretratamiento (no mostrado), indica que el factor con efecto estadístico más significativo sobre la fermentabilidad es la concentración de $\mathrm{NaOH}$, además, que el conjunto de variables experimentales y rangos evaluados explica un $90,27 \%$ de la variabilidad en fermentabilidad.

Los resultados obtenidos, mostrados en la figura 2, exhiben concentraciones de etanol entre 10 y $26 \mathrm{~g} / \mathrm{L}$ correspondientes a rendimientos de 54,7 y $132,0 \mathrm{mg}$ de etanol/g de biomasa seca alimentada respectivamente, para 24 horas de fermentación; sin embargo, esta variabilidad en la concentración de etanol para las diferentes condiciones de pretratamiento también se puede deber a la eficiencia de la hidrólisis enzimática para la transformación de celulosa en azúcares fermentables, lo cual es una consecuencia de las condiciones del pretratamiento; y la disponibilidad que estos azúcares tienen dentro de la suspensión de manera que puedan ser aprovechados por el microorganismo para su transformación en el bioalcohol. En este estudio la concentración más alta de etanol obtenida para pasto elefante de 26,05 g/L, corresponde a condiciones de pretratamiento de $120^{\circ} \mathrm{C}, 2 \%(\mathrm{w} / \mathrm{w})$ de $\mathrm{NaOH}$, relación sólido líquido de 1:20 y 60 minutos de residencia.

Realizando el mismo análisis de los resultados obtenidos para la fermentabilidad del pasto king grass después del proceso de pretratamiento bajo diferentes condiciones de operación, no existe una influencia marcada de alguna de las variables experimentales del pretratamiento que pueda correlacionarse con la producción de etanol obtenida con la producción de azúcares reductores. Por lo tanto la maximización del rendimiento hacia la producción de este bioalcohol depende de las condiciones de las etapas previas de hidrólisis enzimática, lo cual es también evidenciado del análisis estadístico realizado a los datos obtenidos para el diseño experimental planteado (no mostrado), donde el pretratamiento sólo explica un 79,98 \% de la variabilidad de la fermentabilidad, y no hay una correlación significativa entre ésta con ninguno de los factores evaluados: temperatura, concentración de $\mathrm{NaOH}$, relación sólido líquido y tiempo de pretratamiento.

En la figura 2, se pueden observar los resultados de máxima concentración de etanol obtenidas bajo las diferentes condiciones de pretratamiento (tabla 1) de pasto king grass, después de 24 horas de fermentación. Aunque, los resultados no presentan variaciones drásticas con respecto a las condiciones de operación evaluadas en cada uno de los experimentos, se puede notar que con altas temperatura $\left(120^{\circ} \mathrm{C}\right)$ y relación sólido: líquido (1:20 w/w) se obtiene la máxima concentración de etanol de 27,7 g/L correspondiente a un rendimiento de 142,2 $\mathrm{mg}$ de etanol/ $\mathrm{g}$ de biomasa seca alimentada al proceso. 


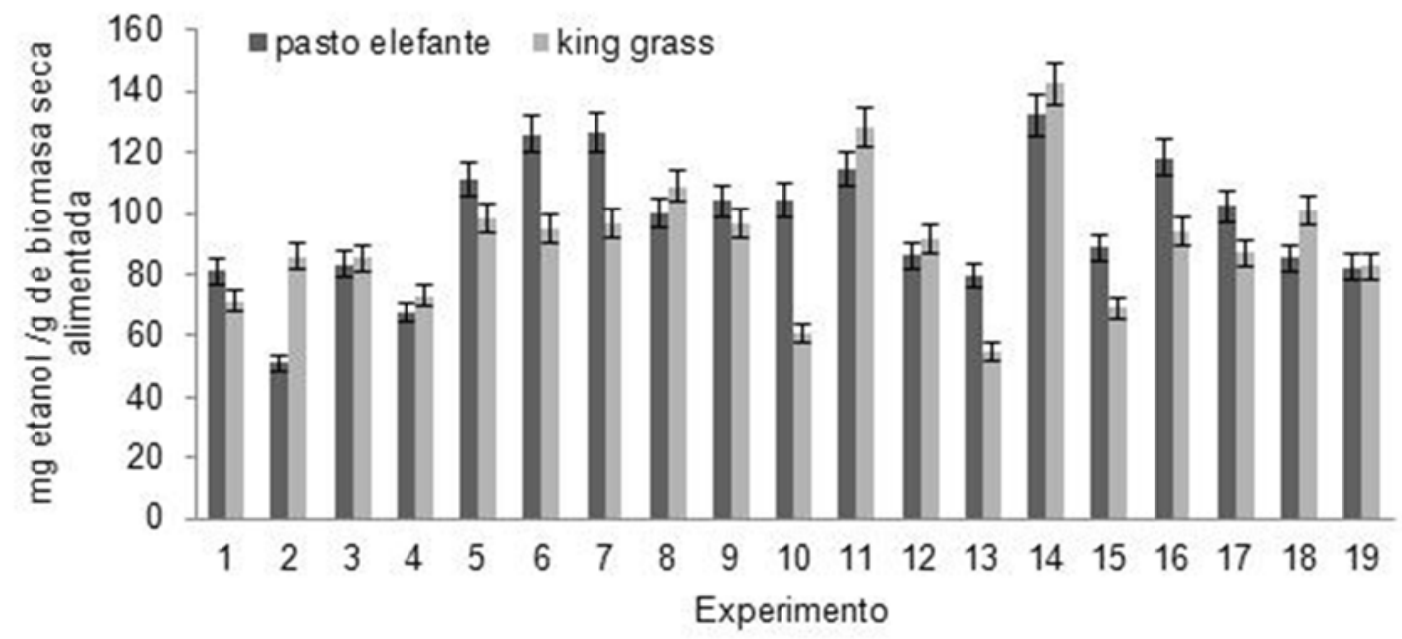

Fig. 2: Concentración de etanol obtenida bajo las diferentes condiciones de pretratamiento del pasto elefante y king grass.

\section{DISCUSIÓN}

Debido a los distintos efectos que tiene sobre la biomasa la deslignificación alcalina con $\mathrm{NaOH}$, como son: i) hinchamiento de la biomasa, lo que conduce a un aumento del área superficial interna, ii) un cambio de la estructura de la celulosa a una forma que es más densa y termodinámicamente más estable que la celulosa original además de disminución de la cristalinidad, iii) separación de las uniones estructurales entre la lignina y los carbohidratos, y una rotura de la estructura de la lignina, y iv) solubilización, redistribución y condensación de la lignina (Hendriks y Zeeman, 2009; Sun y Cheng, 2002); se estudió el pretratamiento de deslignificación con $\mathrm{NaOH}$ con el fin de optimizar las condiciones de pretratamiento que maximizaran la producción de etanol a partir de estas materias primas, mediante el diseño experimental mostrado en la tabla 1. Los resultados obtenidos en esta investigación constituyen un gran aporte en cuanto a las materias primas disponibles para la producción de biocombustibles a gran escala.

Los efectos observados sobre la disminución de lignina en los materiales se debie al efecto que tiene el pretratamiento alcalino de remover o modificar la lignina, favoreciendo la accesibilidad de las enzimas y mejorando la digestibilidad de la celulosa (Wang et al., 2008); sin embargo, la efectividad del pretratamiento alcalino depende de las condiciones de temperatura, tiempo y concentración de $\mathrm{NaOH}$, que pueden llevar a la hidrólisis de la celulosa y hemicelulosa, disminuyendo la cantidad de sólido recuperado para las etapas posteriores de hidrólisis y fermentación. De estos resultados se concluye en cuanto a la remoción de lignina que las mejores condiciones de pretratamiento para el pasto elefante son $100^{\circ} \mathrm{C}, 2$ horas, $0,7 \%(\mathrm{w} / \mathrm{w})$ de $\mathrm{NaOH}$ y una relación sólido a líquido de 1: $17.5(\mathrm{w} / \mathrm{w})$, y para el pasto king grass son $80{ }^{\circ} \mathrm{C}, 3$ horas, $2 \%$ (w/w) $\mathrm{NaOH}$ y una relación sólido a líquido de 1:20 (w/w).

Durante el pretratamiento alcalino la lignina removida puede descomponerse llevando a la formación de compuestos tóxicos para las enzimas y los microorganismos como ácido acético y ácido fórmico, como fue evidenciado por HPLC en las muestras de licor negro obtenidas del pretratamiento de los materiales. De igual manera, durante el pretratamiento alcalino, ocurre la hidrólisis de celulosa y hemicelulosa, llevando a la formación de azúcares monoméricos como glucosa, xilosa y arabinosa, y a la formación de grandes cantidades de ácido acético como producto de la hidrólisis de los grupos acetil, además, la xilosa se degrada posteriormente dando como resultado la formación de furfural, que fue evidenciado en el licor negro del pasto elefante pretratado a temperaturas de $120^{\circ} \mathrm{C}$ y $133.6^{\circ} \mathrm{C}$. Aunque los compuestos formados durante las reacciones de degradación de los azúcares bajo las condiciones de pretratamiento estudiadas pueden afectar el comportamiento de la enzima y el microorganismo durante las etapas de hidrólisis y fermentación, la implementación de una etapa previa de neutralización del material pretratado es muy importante a la hora de mitigar el efecto de estos compuestos inhibidores de la actividad (enzimática y fermentativa), y así mejorar tanto la producción de azúcares reductores como la productividad a etanol; ya que los diferentes estudios existentes reportados muestran que los principales agentes inhibidores son el ácido acético y el furfural cuando sus concentraciones superan los límites permitidos por la enzima o el microorganismo. Particularmente, el furfural puede disminuir la velocidad específica de crecimiento del microorganismo, el rendimiento de biomasa, y el rendimiento específico y volumétrico a etanol (Palmqvist y Hahn, 2000a; Palmqvist et al., 1996; Palmqvist y Hahn, 2000b). 
Los resultados obtenidos en la hidrólisis de pastos elefante y king grass después de diferentes condiciones de pretratamiento alcalino, son similares a muchos de los resultados reportados para pastos en cuanto a la cantidad de azúcares reductores producidos por gramo de biomasa alimentada al proceso. Diferentes estudios sobre la hidrólisis de switchgrass y pasto bermuda costero tratados bajo diferentes condiciones de pretratamiento alcalino, reportan entre 400 y $600 \mathrm{mg}$ de azucares reductores totales/ g de biomasa (Nlewem y Thrash, 2010; Wang et al., 2012; Wang et al., 2010c; Xu y Cheng, 2011). Cabe resaltar además, que el tiempo necesario para lograr una eficiente conversión de la celulosa presente en los pastos en azúcares fermentables, correspondiente a 24 horas de hidrólisis enzimática es mucho menor que el tiempo reportado en muchos estudios de 72, 96 horas y hasta 168 horas de hidrólisis (Alizadeh et al., 2005; Keshwani et al., 2007; Koegel et al., 1997; Wang et al., 2010c).

Las variables más influyentes del pretratamiento son la temperatura, la concentración de $\mathrm{NaOH}$ y la relación sólido líquido, sin embargo, cualquier combinación de estas variables pueden dar rendimientos muy similares en cuanto a la producción etanol. Con los resultados obtenidos, se puede concluir que con unas condiciones de pretratamiento de $120^{\circ} \mathrm{C}$, por 1 hora con una concentración de $\mathrm{NaOH}$ de $2 \%$ (w/w) y una relación sólido a líquido de 1: $20(\mathrm{w} / \mathrm{w})$, se obtienen los más altos rendimientos a etanol correspondientes a $141,0 \mathrm{mg}$ de etanol/g de biomasa y $121,2 \mathrm{mg}$ de etanol/g de biomasa para king grass y pasto elefante, respectivamente, los cuales equivalen a rendimientos máximos del teórico de 94,7\% y 95,07\% como muestra de la eficiencia en el aprovechamiento de la fracción celulósica para la transformación de este tipo de biomasa en etanol. En la literatura se reportan rendimientos de $92 \%$ y $89.2 \%$, para materiales como switchgrass y residuos de maíz (Faga et al., 2010; Isci et al., 2009; Wang et al., 2008), (Shao et al., 2010), y para una especie de pasto elefante sin pretratar se han reportado un rendimiento de $44,2 \%$ con respecto al teórico de xilosa y glucosa (Yasuda et al., 2012). Los rendimientos obtenidos para los pastos elefante y king grass pretratados mediante deslignificación alcalina con $\mathrm{NaOH}$ en este estudio, son comparables con los resultados reportados, además de obtener deslignificaciones hasta de $94,0 \%$ en el caso del pasto king grass.

Por otra parte, los efectos tan variables y diferentes obtenidos en cuanto al efecto de las variables de pretratamiento evaluadas sobre la fermentabilidad de los pastos, se debe principalmente a la composición de estos materiales, por lo cual requieren condiciones diferentes de pretratamiento, e hidrólisis para poder hacer un aprovechamiento eficiente de las fracciones de interés y hacer que el proceso de producción de etanol a partir de estas materias primas sea viable desde el punto de vista técnico, económico y ambiental.

\section{CONCLUSIONES}

Bajo las condiciones evaluadas durante la optimización de la deslignificación alcalina con NaOH para estos materiales, se pudo observar una alta remoción de lignina, 88,4\% y 94,0\% para pasto elefante y pasto king grass respectivamente. En la hidrólisis enzimática se obtuvieron máximos de concentración de azúcares reductores de 515,6 mg/g de biomasa, para pasto elefante y 444,4 mg/ g de biomasa, para pasto king grass. Aunque pudieron lograrse altas remociones de lignina, lo cual es un factor importante para favorecer la hidrólisis enzimática de los materiales, no puede establecerse una relación directa entre los resultados obtenidos y las condiciones evaluadas, esto debido al grado de severidad del pretratamiento que puede lograr efectos diferentes sobre la estructura de los materiales. En este estudio se obtuvieron durante el SSF de los pastos elefante y king grass, concentraciones de etanol de 26,05 g/L y 27,71 g/L, en 24 horas de fermentación, lo cual corresponde a un rendimiento máximo teórico de $94,7 \%$ y 95,07\%, respectivamente, con relación a la celulosa contenida en el material pretratado. Por otra parte, la formación de inhibidores como ácido acético, ácido fórmico y furfural no afecta negativamente la hidrólisis y fermentación de los sustratos, debido que en la etapa previa de destoxificación por neutralización y lavado del material pretratado, pudo haberse logrado una alta remoción de estos compuestos de la fracción sólida, logrando así un alto desempeño de la enzima y el microorganismo empleados. De acuerdo a los resultados obtenidos en este estudio, los pastos elefante y king grass se presentan como materias primas potenciales para la transformación de la fracción celulósica de este tipo de biomasa en etanol.

\section{AGRADECIMIENTOS}

El grupo Procesos Fisicoquímicos Aplicados de la Universidad de Antioquia agradece a Empresas Públicas de Medellín EPM por la financiación del proyecto que enmarca esta investigación.

\section{REFERENCIAS}

Abril, D.R. y A.J. Abril, Etanol lignocelulósico. ¿Aditivo o sustituto para la gasolina?, $V$ Congreso Iberoamericano de investigación en celulosa y papel, Guadalajara, Mexico 20 a 23 de Octubre (2008). 
Alizadeh, H., F. Teymouri, T.I. Gilbert y B.E. Dale, Pretreatment of Switchgrass by Ammonia Fiber Explosion ( AFEX), Appl. Biochem. Biotechnol, 121-124, 1133-1141 (2005).

Alvira, P., M. Ballesteros y M.J. Negro, Pretreatment technologies for an efficient bioethanol production process based on enzymatic hydrolysis : A review, Bioresource Technology, 101(13), 4851-4861 (2010).

Anderson, W.F., Dien, B.S., Brandon, S.K., y J.D. Peterson, Assessment of bermudagrass and bunch grasses as feedstock for conversion to ethanol, Appl. Biochem. Biotechnol, 145(1-3), 13-21 (2008).

Anderson, W.F., J. Peterson, D.E. Akin y W.H. Morrison, Enzyme pretreatment of grass lignocellulose for potential high-value co-products and an improved fermentable substrate, Appl. Biochem. Biotechnol, 121124, 303-310 (2005).

Banerjee, G., S. Car, J.S. Scott-Craig, D.B. Hodge y J.D. Walton, Alkaline peroxide pretreatment of corn stover: effects of biomass, peroxide, and enzyme loading and composition on yields of glucose and xylose, Biotechnology for biofuels, 4(1), 16 (2011).

Brandon, S.K., L.N. Sharma, G.M. Hawkins, W.F. Anderson, C.K. Chambliss y J. Doran-Peterson, Ethanol and co-product generation from pressurized batch hot water pretreated T85 bermudagrass and Merkeron napiergrass using recombinant Escherichia coli as biocatalyst, Biomass Bioenergy, 35(8), 3667-3673 (2011).

Cardona, E., L.A. Rios y J.D. Peña, Disponibilidad de Variedades de Pastos y Forrajes como Potenciales Materiales Lignocelulósicos para la Producción de Bioetanol en Colombia, Inf. tecno, 23, 6, 87-96 (2012).

Digman, M.F., y otros 7 autores, Optimizing on-farm pretreatment of perennial grasses for fuel ethanol production, Bioresour. Technol, 101(14), 5305-14 (2010).

Faga, B.A., M.R. Wilkins y I.M. Banat, Ethanol production through simultaneous saccharification and fermentation of switchgrass using Saccharomyces cerevisiae $D 5 A$ and thermotolerant Kluyveromyces marxianus IMB strains. Bioresour. Technol, 101(7), 2273-2279 (2010).

Galbe, M. y G. Zacchi, Pretreatment of lignocellulosic materials for efficient bioethanol production, Adv. Biochem. Eng./Biotechnol., 108, 41-65 (2007).

Gnansounou, E. Production and use of lignocellulosic bioethanol in Europe: Current situation and perspectives. Bioresour. Technol., 101(13), 4842-50 (2010).

González, I., M. Betancourt, A. Fuenmayor y M. Lugo, Producción y composición química de forrajes de dos especies de pasto Elefante (Pennisetum sp . ) en el Noroccidente de Venezuela. Zootecnia Trop, 29(1), 103-112 (2011).

Guo, G., W. Chen, W. Chen, L. Men y W. Hwang, Characterization of dilute acid pretreatment of silvergrass for ethanol production. Bioresour. Technol., 99, 6046-6053 (2008).

Hendriks, A.T.W.M., y G. Zeeman, Pretreatments to enhance the digestibility of lignocellulosic biomass. Bioresour. Technol., 100, 10-18 (2009).

Isci, A., J.N. Himmelsbach, A.L. Pometto, D.R. Raman y R.P. Anex, Aqueous Ammonia Soaking of Switchgrass Followed by Simultaneous Saccharification and Fermentation. Appl. Biochem. Biotechnol., 144(1), 69-77 (2007).

Isci, A., J.N. Himmelsbach, J. Strohl, A.L. Pometto, D.R. Raman, y R.P. Anex, Pilot-scale fermentation of aqueous-ammonia-soaked switchgrass. Appl. Biochem. Biotech., 157(3), 453-62 (2009).

Keshwani, D.R. y J.J. Cheng, Switchgrass for bioethanol and other value-added applications: A review. Bioresour. Technol., 100(4), 1515-1523 (2009). 
Keshwani, D.R., V. Chiang, J.J. Cheng, J.C. Burns y L. Li, Microwave Pretreatment of Switchgrass to Enhance Enzymatic Hydrolysis, Annual International Meeting Sponsored by ASABE, Minneapolis, Minnesota (2007).

Koegel, R.G., H.K. Sreenath y R.J. Straub, Liquid Hot Water ( LHW ) Pretreatment of Alfalfa Fiber Destined for Ethanol Production, Research Summaries, 25-27 (1997).

López, A.F., G.A. Ortegón y F.A. Robles, Obtaining of Reducing Sugars from Kikuyu Grass ( Pennisetum Clandestinum ). AVANCES Investigación en Ingeniería, 13, 4-7 (2010).

Nlewem, K.C. y M.E. Thrash, Comparison of different pretreatment methods based on residual lignin effect on the enzymatic hydrolysis of switchgrass. Bioresour. Technol., 101(14), 5426-5430 (2010).

Palmqvist, E. y B. Hahn-hagerdal, Fermentation of lignocellulosic hydrolysates. II: inhibitors and mechanisms of inhibition. Bioresour. Technol., 74(1), 25-33. (2000a).

Palmqvist, E, y B. Hahn-hagerdal, Fermentation of lignocellulosic hydrolysates I : inhibition and detoxification, Bioresour. Technol., 74, 17-24 (2000b).

Palmqvist, E., H.H. Gerdal, M. Galbe y G. Zacchi, The effect of water-soluble inhibitors from steampretreated willow on enzymatic hydrolysis and ethanol fermentation. Enzyme Microb. Technol, 19, 470-476 (1996).

Segura S.F., R. Echeverri y A. Mejía, Delignificación selectiva del pasto Pennisetum purpureum $x$ Pennisetum typhoides usando basidiomicetos ligninolíticos. Vitae, 15(1), 41-50 (2008).

Shao, Q. y otros 7 autores, Enzymatic digestibility and ethanol fermentability of AFEX-treated starch-rich lignocellulosics such as corn silage and whole corn plant. Biotechnol., 1-10 (2010).

Sluiter, A., B. Hames, R. Ruiz, C. Scarlata, J. Sluiter y D. Templeton, Determination of Ash in Biomass Laboratory Analytical Procedure (LAP). National Renewable Energy Laboratory (2008a).

Sluiter, A. y otros 8 autores, Determination of Total Solids in Biomass and Total Dissolved Solids in Liquid Process Samples. National Renewable Energy Laboratory (2008b).

Sluiter, A., R. Ruiz, C. Scarlata, J. Sluiter y D. Templeton, Determination of Extractives in Biomass Laboratory Analytical Procedure (LAP). National Renewable Energy Laboratory (2008c).

Sun, Y. y J.J. Cheng, Hydrolysis of lignocellulosic materials for ethanol production: a review. Bioresour. Technol., 96, 1-11. (2002).

Sun, Y. y J.J. Cheng, Dilute acid pretreatment of rye straw and bermudagrass for ethanol production. Bioresour. Technol., 96, 1599-1606 (2005).

Taherzadeh, M.J. y K. Karimi, Pretreatment of Lignocellulosic Wastes to Improve Ethanol and Biogas Production: A Review. Int. J. Mol. Sci., 9, 1621-1651 (2008).

Tran, D.T., C.W. Lin, C.Y. Lai y C.H. Wu, Ethanol Production from Lignocelluloses by Native Strain Klebsiella oxytoca THLC0409. Waste and Biomass Valorization, 2(4), 389-396 (2011).

Wang, Z., D.R. Keshwani, A.P. Redding y J.J. Cheng, Alkaline Pretreatment of Coastal Bermudagrass for Bioethanol Production. Annual International Meeting Sponsored by ASABE. Providence, Rhode Island (2008).

Wang, Z., D.R. Keshwani, A.P. Redding y J.J. Cheng, Sodium hydroxide pretreatment and enzymatic hydrolysis of coastal Bermuda grass, Bioresour. Technol., 101(10), 3583-5 (2010a). 
Wang, Z., R. Li, J. Xu, J.M. Marita, R.D. Hatfield, R. Qu y J.J. Cheng, Sodium hydroxide pretreatment of genetically modified switchgrass for improved enzymatic release of sugars, Bioresour. Technol., 110, 364370 (2012).

Woodward, W.T.W. The Potential of Alfalfa, Switchgrass and Miscanthus As Biofuel Crops in Washington, Energy, 1-7 (2006).

$\mathrm{Xu}, \mathrm{J} . \mathrm{y}$ J.J. Cheng, Pretreatment of switchgrass for sugar production with the combination of sodium hydroxide and lime, Bioresour. Technol., 102(4), 3861-3868 (2011).

Yasuda, M., A. Miura, T. Shiragami, J. Matsumoto, I. Kamei, Y. Ishii y K. Ohta, Ethanol production from nonpretreated napiergrass through a simultaneous saccharification and fermentation process followed by a pentose fermentation with Escherichia coli KO11. J. Biosci. Bioeng., 1-5 (2012).

Yoshida, M., y otros 7 autores, Effects of Cellulose Crystallinity, Hemicellulose, and Lignin on the Enzymatic Hydrolysis of Miscanthus sinensis to Monosaccharides. Biosci., Biotechnol., Biochem., 72(3), 805-810 (2008). 